\title{
DISSOCIATION AS A GUIDE TO DEVELOPMENTAL ASSESSMENT
}

\author{
BY \\ R. S. ILLINGWORTH \\ From the Children's Hospital, Sheffield, and the Department of Child Health, the University of Sheffield
}

(RECEIVED FOR PUBLICATION SEPTEMBER 18, 1957)

In the assessment of an infant's development, in the light of the mother's developmental history and of one's own developmental examination, one considers not only how far one's findings tally with the mother's history, but the relationship of one field of development to another. For instance, if a child's speech development is quite out of step with his motor development, an attempt to determine the reason for it and its significance must be made. Such a departure from the usual relationship of one field of development to another may be termed 'dissociation'. It is a common and important finding which has received little or no attention in the literature. For convenience I shall consider it in different fields of development.

\section{Delayed Motor Development Without Retardation in Other Fields}

The commonest cause of delayed motor development in an infant is mental deficiency; but in mental deficiency there will also be retardation in other fields of development such as social behaviour, interest in surroundings, and speech, for no child with isolated delay in motor development can be mentally defective.

Obvious causes of isolated motor retardation are hypotonia and hypertonia. Rare causes include infantile myopathies, diastematomyelia and other purely mechanical defects.

Hypotonia. The causes of hypotonia have been reviewed by Walton $(1956,1957)$ and need not be recapitulated here. They include amyotonia congenita and allied conditions. Hypotonia sufficient to cause significant motor retardation could hardly be missed. In the developmental history and examination there is a striking dissociation between the grossly retarded motor development and the normal social behaviour. For instance, the child has begun to smile at the usual age (4 to 6 weeks), to vocalize his pleasure two or three weeks later, to take a normal interest in his surroundings, to follow his mother's movements with his eyes (at 2 or 3 months of age), and to turn his head to sound at about 3 months. Later on he has shown excitement at feed times, and has imitated his parents in sounds and simple movements (6 to 9 months). His vocalizations have followed the usual pattern and his speech has developed at the usual time, so that he says three or four words with meaning by 12 months, and has joined two or three words together by 21 to 24 months. On the other hand, he is grossly retarded in head control, and subsequently in sitting, creeping and walking. An illustrative case history is as follows:

When this girl was seen at 13 months her head control was that of a 10-weeks-old baby. The manipulative development was average. She was saying single words with meaning. There was generalized hypotonia, and as the knee jerks were present the diagnosis of congenital hypotonia was made. Subsequently she put words together at 27 months and walked without help at 5 years.

This child has a mild congenital hypotonia with an average intelligence. The normal social and subsequently normal speech behaviour would preclude the suspicion of mental deficiency.

Less easy to assess is the combination of mental deficiency with hypotonia. This is not a particularly common combination, but the complication of hypotonia might well lead to too gloomy a prognosis for intellectual development because of the severe motor retardation. One should look for a glimmer of hope in the relationship of the motor defect to other fields of development. In uncomplicated mental deficiency there tends to be relatively less retardation in motor development than in social behaviour and speech. When there is an additional mechanical difficulty, such as cerebral palsy, that position is reversed. One might find, therefore, that there is very severe motor retardation with only moderate retardation in speech. This would indicate a motor defect with an only moderately low intelli- 
gence quotient (I.Q.). The case of cerebral palsy described below is an example.

Acrodynia (pink disease) may present a confusing picture, in that the mother's history indicates that early development was normal, and that deterioration in motor development then ensued.

An 8-month-old child was referred for confirmation of the diagnosis of mental defectiveness. The mother was not able to give a reliable developmental history. The child's head control (when placed in the prone and when pulled to the sitting position) was that of a 4-monthold baby, but he was interested in test objects. His manipulative development was not less than that of an average 8-month-old baby. His grasp of a 1-in. cube was a mature one, with a rapid transfer to the other hand. He was seen to chew. He was markedly hypotonic and other signs of acrodynia were found. He made an uninterrupted recovery and became a normal child.

The dissociation between the delayed motor and normal manipulative and social behaviour showed immediately that this boy could not be mentally defective, but that there should be a mechanical difficulty affecting his gross motor development.

Rickets, which is now hardly ever seen in this country, would give a similar developmental picture, because of the hypotonia with which it is associated.

Severe illness may cause delay in motor development because of the poor muscle tone which it induces while development in other fields is likely to be normal.

Hypertonia. Cerebral palsy presents considerable developmental problems because it is seen in several different forms; there are all degrees of severity, and it is so frequently associated with a low level of intelligence which itself greatly affects the child's development.

The diagnosis of spastic paraplegia can often be made in the older infant on the history alone, provided that the intelligence is average. There is a story of normal social development (smiling by the age of 6 weeks, and so on), with normal manipulative development (voluntary grasping by 5 months), normal speech, but grossly delayed walking. The age of sitting may or may not be normal. The mother may have noticed stiffness of the lower limbs. The diagnosis is confirmed by the physical examination. The following history is an example:

A girl grasped objects voluntarily at 5 months, sat unsupported at 8 months, was helping her mother to dress her at 13 months (by holding her arm out for clothes and the foot out for a shoe) and spoke in sentences at 23 months. She was not able to walk at 5 years. She had a paraplegia with a normal I.Q. (The school medical officer assessed her on the Terman-Merrill scale as 'average' at 5 years without giving a figure.)

A mild degree of paraplegia can easily be missed in the early months.

I observed a child from 10 months of age on account of defective head control. There was no retardation in other fields. I suspected paraplegia, but subsequently decided that the knee jerks were within normal limits. There was no adductor spasm on abducting the hips. I saw the boy repeatedly, and eventually when he was 4 years old found undoubted evidence of mild disease of the pyramidal tracts involving the lower limbs. There was no deformity. He had learned to sit without support at 14 months, to feed himself with a cup at 18 months, and to join words together at 23 months. He was not able to walk at 4 years. At that time his developmental age in fields other than walking was 40 months.

Unfortunately, an average level of intelligence is unusual in cerebral palsy, so that one has the confusing picture of two factors combining to affect and retard development. Here the phenomenon of dissociation is of real value in developmental prognosis. It is particularly important to assess the child's interest in surroundings, his powers of concentration and speech. Whereas a mentally defective spastic child will make little effort to grasp an object, a spastic child of the same age and degree of spasticity but with a good level of intelligence will make determined efforts to grasp. Hence one notes the dissociation between the physical disability and the powers of concentration. If retardation in speech is only slight, severe mental deficiency is impossible: hence it is a most important indication to look for in assessing a child with cerebral palsy. Naturally speech is often retarded by other factors, such as deafness, in the presence of a good level of intelligence, so that no absolute reliance can be placed on the absence of speech as an indication of low intelligence.

A 24-month-old child was referred with a diagnosis of mental deficiency. He began to grasp objects voluntarily at 10 months, but was not able to sit. He began to say words with meaning at 18 months and to join words together at 30 months.

This gross motor delay with only a moderate delay in speech would suggest a severe mechanical disability with only a moderately low I.Q. He had a spastic quadriplegia, and his I.Q. at 5 years was 71 .

A possibility to consider on finding isolated motor retardation is the subsequent development of athetoid movements, indicating that the child is in fact suffering from athetosis. It is by no means always the case, however, that motor retardation is found in early infancy in children who will subsequently be shown to be athetoids, for, provided that the I.Q. is average, the motor development in 
some of these children is normal. One would certainly bear the possibility in mind if there were a history of severe perinatal anoxia, of improperly treated haemolytic disease of the newborn or hyperbilirubinaemia with prematurity.

Other Causes. There remain some cases of isolated motor retardation in which even prolonged observation fails to reveal any sign of organic disease.

A baby, aged 7 months, was referred by a paediatrician because of defective head control. He had been examined with regard to suitability for adoption. On examination his head control (when prone and when pulled to the sitting position) was that of a 3-month-old baby, but his manipulative development was that of a 7-8 month baby. His interest in surroundings and his alertness seemed average. He was able to chew. There were no signs of cerebral palsy. I expressed the opinion that this was merely isolated motor retardation. His subsequent history showed that he spoke single words and sat without support at 1 year, put words together at 18 months and walked without help at 23 months. Subsequent examination showed that he was normal in all respects, with an I.Q. of just over 100 .

In view of his prospective adoption, the responsibility of making a correct diagnosis was a big one.

\section{Normal Motor Development with Retardation in Other Fields}

One occasionally sees a child in whom motor development is within 'normal' limits but in whom defective social and speech development leads to a diagnosis of mental deficiency. It is not rare to see relatively advanced motor development in mongols in early infancy-a fact noted by Gesell.

I observed a mongol who began to smile at 4 months, to grasp objects voluntarily at 6 months, and to sit unsupported at $7 \frac{1}{2}$ months. At this time his interest in his surroundings was very defective. Development then slowed down, and he only began to walk without help at 34 months and to put words together at 69 months. At this age his I.Q. was 28. I saw another mongol who at 8 months was sitting securely without support, quite as well as an average baby; but his interest and concentration were notably defective, and he showed hand regard-normally lost by 16 weeks. His I.Q. subsequently was 30 .

A girl sat without support at $7 \frac{1}{2}$ months, and walked without help at 21 months. Concentration was poor. She was only able to say six words at 39 months. At five years her I.Q. was 50. Another girl sat without support at 8 months, and walked without help at 17 months, but there was no speech and concentration was poor. There was full sphincter control by 24 months. She began to say single words at 3 years and to put words together at 5. At ten years her I.Q. was 20. Another girl sat without support at 6 months, and walked without help at 18 months, but I was impressed by the aimless hyperkinesis, defective concentration, and delayed speech development. Her I.Q. at 56 months was 52 . In none of these cases was there any degenerative disease of the nervous system or deterioration in performance.

\section{Other Variations in Motor Development}

One sometimes sees the picture of a 'slow-starter' - a child who is seriously retarded in motor development in the first few weeks and then catches up.

A child with a birth weight of $5,000 \mathrm{~g}$. and a normal perinatal history had a complete head lag at 13 weeks like that of a newborn baby and did not follow with the eyes till 17 weeks or smile till 18 weeks. From that time she made rapid strides in development, and at 25 weeks her head control was that of a 16-week-old baby. She sat without support at $7 \frac{1}{2}$ months, and walked without support at 1 year. She was able to say ten words with meaning at that age. Her I.Q. at 5 years was 122 .

Some children develop normally for the first few weeks and then develop thyroid deficiency, encephalitis, or signs of a degenerative disease of the nervous system. When these develop suddenly, as in the development of akinetic seizures with hypsarrhythmia (Illingworth, 1955), one sees, shortly after the change has occurred, the unusual picture of normal motor development with grossly defective concentration and interest in surroundings, indicating severe mental deficiency.

Within the field of motor development itself there are occasionally certain unusual variations. Normally a child learns to sit before he creeps. I have seen the reverse happen in a child who had been brought up in an institution, and who presumably had never been given any chance to sit. One commonly sees dissociation between the sitting and standing development: for instance, an 8-month-old child is seen to sit securely, like an average child of the same age, and yet to bear as little weight on the legs as a 2- or 3-month-old child. This is simply due to the mother not allowing him to bear weight on the legs in the erroneous belief that it will weaken or otherwise harm them.

Sometimes the age of sitting unsupported is quite average, while the child is unusually late in walking without help. In all other fields the child develops normally. For instance, a child sits unsupported at 7 months, but cannot walk without help till 18 months or later. As has already been stated, one sees this in cases of paraplegia. In most children, however, there is no organic disease. One factor may be lack of confidence or fear of falls. When a child refuses to walk without help for a long period (for four or five months, for example), simple 
observation will show that his walking is maturing, and that as soon as he decides to walk alone he is walking well, like a child who has achieved the skill several weeks earlier.

Unusual but isolated advancement in motor development certainly does not indicate a high level of intelligence.

I saw a child sit without support at 4 months, creep (not crawl) at $4 \frac{1}{2}$ months, and pull himself to the standing position at 6 months. I expressed the opinion at that time that this was merely an isolated advancement, and that his I.Q. would not prove to be above the average. This was because he was in no way above the average in other fields of development. Although he subsequently walked without help at 8 months, his I.Q. at 5 years was 88 . I saw another boy who walked unaided at 8 months, but was not advanced in other fields. His I.Q. at 6 years was 103.

Sphincter Control. Sphincter control is an unimportant field of development from the point of view of assessing a child's intelligence. It is delayed by a variety of organic and semi-organic conditions, and in particular by over-enthusiastic toilet training. In addition, there are very strong familial factors, unrelated to intelligence. When a child with average development in other fields is very late in acquiring control of the bladder, there is nearly always a family history of the same complaint. In mentally defective children one occasionally finds that sphincter control has been acquired at the usual time.

I would pay little attention to a history of unusually early acquisition of control, unless I were really satisfied that the control is truly voluntary, in that the child definitely asks for the chamber when he wants to void. It is easy to confuse the early 'conditioning', which is quite involuntary, with voluntary control.

Eyes, Ears, Manipulation. Unusual retardation in the use of the eyes, ears and hands, in the absence of retardation in other fields, is very rare. It may occur in the presence of obvious physical defectsvisual or auditory in the case of the special senses, or of myotonia or other mechanical disability in the case of the hands. Unusual advancement in these fields is of no prognostic importance, though special manipulative aptitude can sometimes be detected in the early months.

Chewing. I find that the age of beginning to chew (average 6 months) is a useful milestone of development, with little variation in children of average development, and invariable retardation in mental deficiency.
I know of only one variation from the average in children of normal development in other fields, and that is related to mismanagement, in the form of failure to give the baby solid foods at an age when he should be given them. When they are introduced some months later (at the peak of the period of negativism), refusal to chew may occur.

Social Behaviour. Isolated lateness in social responsiveness, such as smiling and taking notice, is extremely rare in early infancy. It could be due to a visual defect.

In older infants and young children it is not so rare. I saw a child who was suspected of mental deficiency, because of a complete failure to smile or show facial responsiveness, but found that she was an example of congenital facial diplegia (Möbius syndrome). A child first seen by me at the age of 4 years, and in whom the diagnosis was infantile autism, was said by his mother never to have shown any social responsiveness at all. Development in all other respects had been normal. His I.Q. was average. One sees children with relatively 'normal' development, but with defective concentration and interest (see above). This nearly always signifies mental deficiency.

Unusually advanced social development is a good sign of superior intelligence. It is possible that early smiling might be in part related to a child's pleasant personality, but there is no evidence to that effect. I have seen two children smiling in response to social overture in the first week, and vocalizing in the third week: they had I.Q.'s subsequently in the region of 140 .

In later months, unusually good concentration, alertness, determination and interest in surroundings is an important sign of superior intelligence and much more important than advanced motor development. It is unfortunate that it is so difficult to assess it and convert the assessment into figures.

Speech Development. Delayed speech development in the absence of retardation in other fields is never due to mental deficiency. It may occur in children with average or superior intelligence. In the majority of such cases there is either a family history of similar lateness, or there is no discoverable cause. Einstein gave his parents grounds for concern because of his defective speech at the age of 4 years. He was still speaking indistinctly at the age of nine. Other causes of lateness in speech without retardation in other fields include deafness, high-frequency or otherwise. It is never due to 'laziness', and serious harm is done by advising parents to do nothing for such a child unless he 
speaks properly. He does not speak because he cannot, not because he is too lazy to speak. I firmly believe that the statement repeated from textbook to textbook that delay in speech is due to parents and siblings doing things for a child before he asks for them is a myth. I very much doubt the truth of another oft-repeated statement, that lateness in speech is due to jealousy. All children are jealous, and as lateness in speech is common, it is indeed difficult to prove that lateness in speech is due to jealousy and not to something else. I would only go so far as to say that inability to speak may perhaps be a very rare result of jealousy, but that it cannot be proved. Lateness in speech is never due to 'tongue tie' or to cleft palate, though cleft palate may be associated with a low I.Q. and so with lateness in speech. In this case there would be retardation in other fields too.

Unusual advancement in speech is a reliable sign of superior intelligence. I have never seen it in a child of average intelligence.

The importance of relating the level of speech development to that of development in other fields has already been noted.

\section{Summary and Conclusions}

In assessing the development of an infant or young child one should always note the relationship of one milestone of development to another, in order to assess the rate of development, and the relationship of milestones in one field of development to those in another. Not infrequently development in one field is out of step with that in another. This might be termed dissociation. The reasons for this imbalance are discussed.

Isolated delay in motor development may occur without apparent reason, so that the child grows up to be perfectly normal. More often it results from a physical disability, in particular hypotonia or hypertonia. It never signifies mental deficiency.
Relative advancement in motor development in relation to other fields occasionally occurs in mentally defective children. Unusual and isolated advancement in motor development does not mean superior intelligence.

Retardation in social behaviour is of considerable prognostic significance, implying a poor prognosis for intelligence. The degree of retardation in a child with retardation in other fields is of great importance: only slight social retardation with severe motor retardation may occur in the presence of a severe mechanical disability, such as cerebral palsy, with an only slightly lowered intelligence. Advanced social development is a good index of superior intelligence, except that it is difficult to assess in figures.

Isolated delay in speech frequently occurs in children of normal or superior intelligence, and never means mental deficiency. Only moderate retardation in speech in the presence of severe retardation in other fields (as in cerebral palsy: excludes severe mental deficiency. Isolated advancement in speech is a good sign of superior intelligence.

Isolated delay in sphincter control is commonly associated with mismanagement or with familial factors, and is of no importance in developmental assessment. Advancement, relative or absolute, is of little value because of the difficulty in assessing its significance (i.e. whether the control is really voluntary and not mere conditioning).

Delay in chewing may result from mismanagement, and otherwise does not occur alone.

I wish to thank Arnold Gesell, who gave me the opportunity to learn about normal development in his Department.

\section{REFERENCES}

Illingworth, R. S. (1955). Arch. Dis. Childh., 30, 529. Walton, J. N. (1956). Lancet, 1, 1023. 